\title{
Successful Treatment With Baricitinib in a Patient With Refractory Eosinophilic Fasciitis
}

Rahul Sehgal(1), Assistant Professor of Medicine, MBBS, MD; Floranne C. Ernste (D), Associate Professor of Medicine, MD; Sara Eckloff, MD, Department of Medicine, Division of Rheumatology, Mayo Clinic, Rochester, Minnesota, USA. Address correspondence to Dr. R. Sehgal, 1400 Bellinger Street, Eau Claire, WI 54701, USA. Email: sehgal.rahul@mayo.edu. The authors confirm that the authors have no financial disclosures and conflict of interest. This study has not received any financial support or other benefits from commercial sources for the work reported on in the manuscript. Institutional review board approval was not required according to the regulations of the authors' institution. Patient's written informed consent was obtained to publish the material in manuscript.

Eosinophilic fasciitis (EF), or Shulman disease, is a rare sclerosing skin disorder ${ }^{1}$ treated with corticosteroids (CS) and additional immunosuppressive agents ${ }^{2}$.

A 37-year-old man was diagnosed with EF by full-thickness skin biopsy. Complete blood count revealed absolute eosinophil count of 2800. Peau d'orange was present with a "groove sign" on raising forearms. Initial treatment comprised intravenous (IV) methylprednisolone $(1000 \mathrm{mg} /$ day $\times 3$ days $)$ followed by prednisone (60 mg) and methotrexate (MTX) $25 \mathrm{mg}$ weekly for 3 months. Mycophenolate mofetil $2 \mathrm{~g}$ /day was added to MTX for an additional 3 months followed by monthly IV $\operatorname{Ig}(2 \mathrm{~g} / \mathrm{kg})$ for 3 months without improvement.

Based on previous reports of success in treatment of EF with use of Janus kinase (JAK) inhibitors ${ }^{3,4}$ and an ongoing trial evaluating their role in systemic sclerosis (SSc; ClinicalTrials.gov: NCT03274076), we used baricitinib as a safer and cost-effective treatment in our patient with refractory EF. Baricitinib allowed for reduction in corticosteroids, improvement in Health Assessment Questionnaire II score from 2.5 to 1.0, reduction in skin induration, and improvement in elasticity. Three months later, he had considerable improvement in symptoms and skin appearance (Figure 1). We used point-of-care ultrasound to follow the disease course (Figure 2). EF is included in a spectrum of SSc-like disorders, and several cytokines including Th1/ interleukin (IL) 12, Th17/IL-23, 6 (IL-23, IL-6), and transforming growth factor $\beta 1$ pathways play a major role in its pathogenesis $^{5}$. These cytokines transmit signals through JAK. To our knowledge, this is the first case of EF treated successfully with baricitinib and supports growing interest in use of JAK inhibitors in sclerosing skin disorders.

Figure 2. Ultrasound of (upper/medial) right calf (A) performed on General Electric LogiQ E9 before baricitinib treatment showing thickened and heterogeneous superficial gastrocnemius fascia measuring $\sim 2 \mathrm{~mm}$, and subcutaneous tissue edema is present (arrows); and (B) performed on General Electric LogiQ E10 post-baricitinib treatment, showing superficial gastrocnemius fascia thickness measuring $\sim 1 \mathrm{~mm}$ that appears uniform in echogenicity.
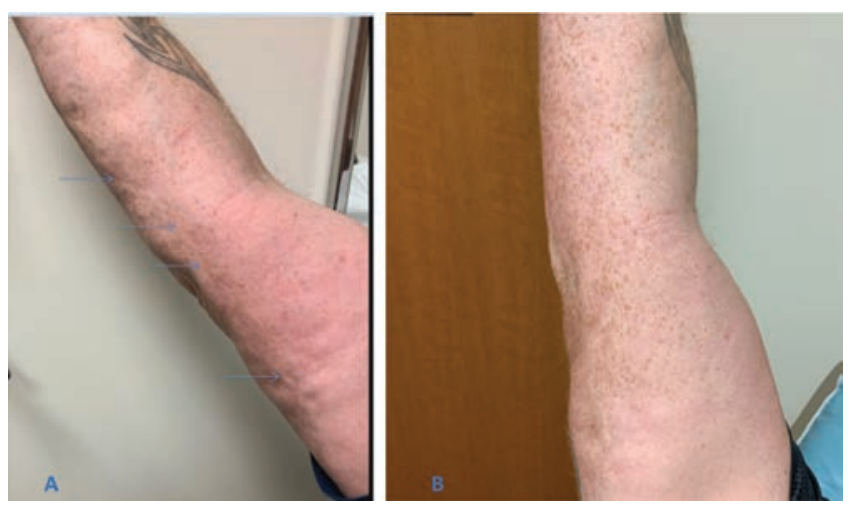

Figure 1. (A) Linear depressions (Groove sign) on the right forearm (arrows) and peau d'orange changes before baricitinib. (B) Post-baricitinib improvement in right forearm linear depressions and skin appears smooth.
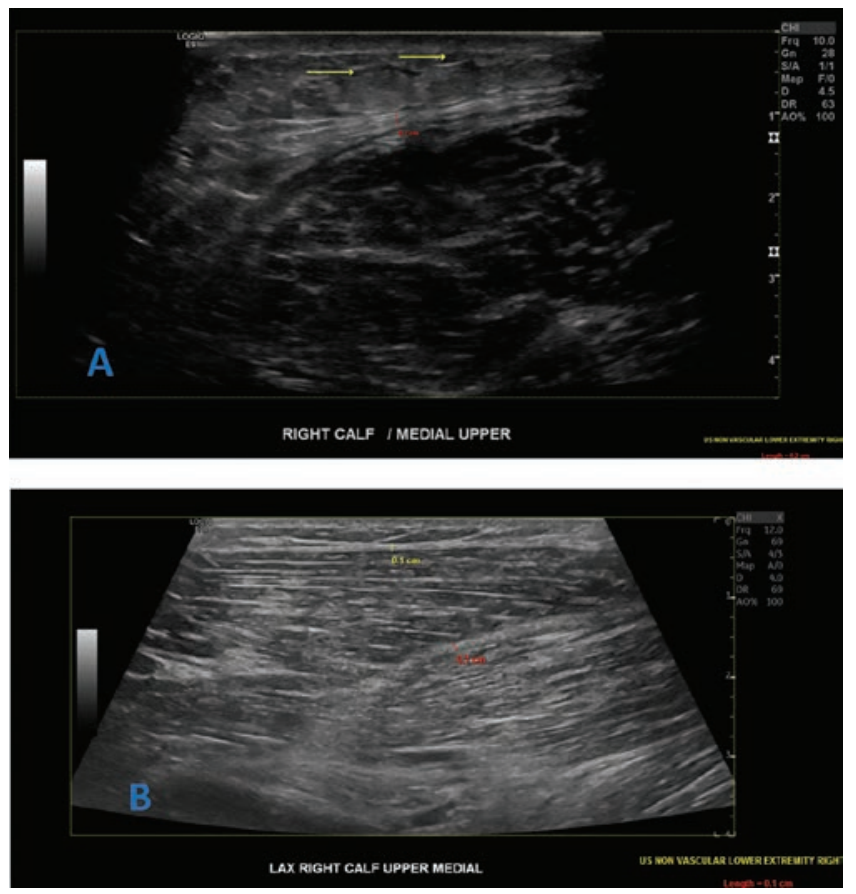


\section{REFERENCES}

1. Shulman LE. Diffuse fasciitis with hypergammaglobulinemia and eosinophilia: a new syndrome? J Rheumatol 1984;11:569-70.

2. Lebeaux D, Francès $C$, Barete $S$, Wechsler B, Dubourg O, Renoux J, et al. Eosinophilic fasciitis (Shulman disease): new insights into the therapeutic management from a series of 34 patients. Rheumatology 2012;51:557-61.

3. Kim SR, Charos A, Damsky W, Heald P, Girardi M, King BA.
Treatment of generalized deep morphea and eosinophilic fasciitis with the Janus kinase inhibitor tofacitinib. JAAD Case Rep 2018;4:443-45.

4. Cao XY, Zhao JL, Hou Y, Wang FD, Lu ZH. Janus kinase inhibitor tofacitinib is a potential therapeutic option for refractory eosinophilic fasciitis. Clin Exp Rheumatol 2020;38:567-8.

5. Mori Y, Kahari VM, Varga J. Scleroderma-like cutaneous syndromes. Curr Rheumatol Rep 2002;4:113-22. 